\title{
A Rapid Fluorometric Test for Aflatoxins in Grains and Raw Peanuts
}

\author{
John L. Richard
}

\begin{abstract}
A rapid method for the fluorometric analysis of aflatoxins in corn, corn meal, popcorn, rice, wheat, cottonseed and peanuts was developed. This method proved to be quantitative, inexpensive and very efficient. Ground samples of the commodity being tested are extracted with methanol-water $(80+20)$ and cleaned up by passage through a solid-phase separatory column. Five hundred $\mu \mathrm{l}$ of purified extract is derivatized with a bromine reagent and the fluorescence of the solution is quantified with a calibrated fluorometer. This method will quantify aflatoxin from 5 to $5000 \mathrm{ppb}$ without dilution and was linear when applied to samples of noncontaminated corn spiked at 0 to $5000 \mathrm{ng}$ aflatoxin $\mathrm{B}_{1} / \mathrm{g}$. Correlation coefficients of the method with HPLC for multiple analyses for corn $(n=34)$, cottonseed $(n=$ 32 ) and peanuts $(n=11)$ were $0.999,0.995$ and 0.980 , respectively. Individual analyses can be conducted in less than $5 \mathrm{~min}$ and grouping of samples is unnecessary. The sensitivity of the method for corn is $5 \mathrm{ppb}$ and the fluorometer, operating under the prescribed conditions, has a limit of detection of $0.6 \mathrm{ng}$ of aflatoxin $\mathrm{B}_{1}$.
\end{abstract}

Key words : fluorometry, aflatoxins, analysis, grains, mycotoxins

The aflatoxins are important hepatotoxic mycotoxins and belong to a chemically related group of compounds called difurocoumarolactones,. The most commonly occurring and most potent aflatoxin is aflatoxin $B_{1}$ and is recognized as being a probable human carcinogen by the International Agency for Research on Cancer ${ }^{1)}$. The occurrence of aflatoxins in food is unavoidable and periodic high level and high incidence contamination occurs in crops in various areas of the world dependent upon the environmental conditions during the growing season. Thus, there is need for accurate analytical procedures to measure the concentration of toxic levels of aflatoxins in these commodities. The major methods of analysis of aflatoxins include TLC, HPLC, ELISA and GC-MS ${ }^{2}$. The TLC of aflatoxins, while a powerful method, lacks the sensitivity and accuracy of quantification of the other methods. The HPLC methods for aflatoxin are the most often used reference method for quantitation of these mycotoxins but the length of time to perform the analysis and the equipment cost is often a limiting factor in the choice of this procedure. The ELISA methods, while being quicker, usually around 20-30 min, sometimes are less specific than other methods. To limit the expense of the ELISA test, samples need to be grouped for analysis. The GC-MS methods are expensive because of the equipment necessary and are lengthy to perform and not suitable for field-testing. Affinity column chromatography followed by fluorometry is quite useful but column shelf life limits the effective time that the

Romer ${ }^{\mathrm{TM}}$ Labs, Inc., 1301 Stylemaster Drive, Union, MO 63084-1156 USA

Fax : +1-636-583-6553 
kit is useful. Therefore, the need for a safe, rapid, accurate and sensitive method for analysis of aflatoxins that can be applied inexpensively to individual samples still exists.

This report describes the use of a solid-phase extraction cleanup column followed by a fluorometric analysis for determination of aflatoxins in grains. This quantitative method has proven to be rapid, accurate (similar to LC) from 5 to $5000 \mathrm{ppb}$ with no dilutions, can be applied to individual samples, is inexpensive and is GIPSA approved.

\section{Materials and Methods}

\section{Sample Preparation and Extraction}

Prior to extraction, representative samples of grain (5 lbs of corn or $3 \mathrm{lbs}$ of sorghum) were ground with the Romer ${ }^{\mathrm{TM}}$ Series II Mill (Romer ${ }^{\mathrm{TM}}$ Labs, Inc., Union, MO). Cottonseed was ground with the RAS mill (Romer ${ }^{\mathrm{TM}}$ Labs, Inc., Union, MO) and peanuts were obtained, already ground, as a peanut paste. A fifty gm subsample of each commodity sample was placed in a blender jar (one half pint suitable for use with blender) along with $100 \mathrm{ml}$ of reagent grade methanol: water $(80+20)$ (Mallinkrodt Chemical Works, St. Louis, MO). The extraction/blending was conducted for $1 \mathrm{~min}$ on high-speed option with the blender (Osterizer 10 speed blender, Sunbeam-Oster Household Products, Schaumburg, IL). The extraction solvent was decanted into a glass container through filter paper (Whatman \# 4 or equivalent).

\section{Cleanup}

The cleanup of samples was accomplished with one of two columns. The SolSep \# 2001 columns (Romer ${ }^{\mathrm{TM}}$ Labs, Inc., Union, MO), was used for all commodities except cottonseed for which SolSep \# 2002 column (Romer ${ }^{\mathrm{TM}}$ Labs, Inc., Union, MO) is necessary. With the clear plastic tip cap pushed firmly onto the bottom of the AflaSep column, $1000 \mu \mathrm{L}$ of the extract filtrate was placed on the top of the column along with $1000 \mu \mathrm{L}$ of reagent grade acetonitrile. The cap was placed on the column top and mixed thoroughly by hand shaking for $5 \mathrm{sec}$. After the tip cap and column top cap was removed, the syringe adapter (a $20 \mathrm{ml}$ syringe fittled with a \# 1 stopper on the tip for applying pressure to the AflaSep column) was placed on the column and the mixture was slowly (about $30 \mathrm{sec}$ ) pushed through the column into a $12 \times 75 \mathrm{~mm}$ cuvette (Kimble Glass, Vineland, $\mathrm{NJ}$ ) capable of being fitted with a Uni-Flex safety cap $(12 \times 13 \mathrm{~mm}$, Bio Plas, Inc., San Francisco, CA).

\section{Derivatization}

For increasing the fluorescence of the aflatoxins a developer concentrate was made consisting of $0.1 \%$ bromine, (Fisher Scientific, Fair Lawn, NJ) in deionized water. The concentrate was kept in $1 \mathrm{~mL}$ aliquots sealed in amber ampoules. For use in the test a developer working reagent was made by diluting the contents of one ampoule of concentrate $1: 50$ in deionized water in an airtight, amber dispensing container suitable for dispensing 1 $\mathrm{mL}$ volumes.

Five hundred $\mu \mathrm{L}$ of cleaned up extract was placed in a clean $12 \times 75 \mathrm{~mm}$ cuvette, $1000 \mu \mathrm{L}$ of developer working reagent was added, the cuvette was capped and mixed thoroughly with a vortex mixer (Model G-560, Scientific Industries, Inc., Bohemia, NY) for 5 sec. After wiping the cuvette with lint free paper, the cuvette was placed immediately into a calibrated fluorometer. 


\section{Fluorometry}

The standards used for fluorometry were $5.0 \mu \mathrm{g} / \mathrm{mL}$ total aflatoxins $-B_{1}, B_{2}, G_{1}, G_{2}(7 /$ $1 / 3 / 1$ ) in acetonitrile. The fluorometer (Model FX-100, Romer ${ }^{\mathrm{TM}}$ Labs, Inc., Union, MO, equipped with a broad wavelength pulsed Xenon lamp, selected source filters of $365-380 \mathrm{~nm}$ for excitation, and 450-455 nm for emission, a silicon detector and an RS-232C output for printer) was calibrated before use with the high and low calibrators. The base solution for preparing the calibrators was quinine at a concentration of $1 \mu \mathrm{g} / \mathrm{mL}$ in aqueous $0.1 \mathrm{~N} \mathrm{H}_{2} \mathrm{SO}_{4}$. The low calibrator was $0.38 \mathrm{~mL}$ base solution in $200 \mathrm{~mL}$ of aqueous $0.1 \mathrm{~N} \mathrm{H}_{2} \mathrm{SO}_{4}$ to yield 0 . $0019 \mu \mathrm{g}$ quinine $/ \mathrm{mL}$. This calibrator fluorescence is equivalent to the innate background fluorescence of corn. The high calibrator was $0.254 \mathrm{~mL}$ of base solution in $200 \mathrm{~mL}$ of aqueous $0.1 \mathrm{~N} \mathrm{H}_{2} \mathrm{SO}_{4}$ to yield $0.0127 \mu \mathrm{g}$ quinine/mL. This calibrator fluorescence is equivalent to the combined background fluorescence and aflatoxin fluorescence when the corn was spiked at $40 \mathrm{ppb}$. The control calibrator was $1.525 \mathrm{~mL}$ of aqueous $0.1 \mathrm{~N} \mathrm{H}_{2} \mathrm{SO}_{4}$ to yield $0.007625 \mu \mathrm{g}$ quinine $/ \mathrm{mL}$. These calibrators were used as the basis for factor adjustment of the fluorometer for all other commodities for which the test is used. All calibrators are placed in glass, sealed ampoules for reading in the fluorometer. All calibrators are commercially available (Romer ${ }^{\mathrm{TM}}$ Labs, Inc., Union, MO).

To assure the accuracy of the fluorometer after calibration, the control calibrator was placed in it and the value recorded in ppb. The fluorometer is equipped with a program specifically for aflatoxin in various commodities. Therefore, the control calibrator must fall within the specific range (22-26 ppb) as indicated. Once a sample has been inserted into the fluorometer, a $40 \mathrm{sec}$ delay occurs before the result will appear on the screen and be printed.

Commodities and products that have been analyzed using this fluorometric procedure include corn, popcorn, rice, wheat, peanuts, cottonseed and sorghum.

Comparisons with LC analysis were conducted on naturally contaminated samples of corn, cottonseed and peanut paste.

\section{LC Analysis}

The standard used for LC analysis was $5 \mu \mathrm{g} / \mathrm{mL}$ total aflatoxins $-B_{1}, B_{2}, G_{1}, G_{2}(4 / 1 / 4$ / 1 ) in acetonitrile. All solvents used in LC were LC grade (Mallinkrodt Chemical Works, St. Louis, MO).

The LC method was adapted from that described by Kok, et al., ${ }^{3)}$ whereby the Kobra Cell was used for converting bromide ion included in the mobile phase to bromine for postcolumn derivitization of aflatoxin. A portion $(100 \mu \mathrm{L})$ of the sample extract that had passed through the SolSep column for fluorometry was diluted with $440 \mu \mathrm{L}$ of deionized water, mixed and $100 \mu \mathrm{L}$ of this solution was injected into the LC (Shimadzu LC-600 with a Sil-9A autoinjector, RF 535 detector at $360 \mathrm{~nm}$ excitation and $440 \mathrm{~nm}$ emission equipped with a Kobra Cell, and a $4.6 \mathrm{~mm} \times 10 \mathrm{~cm}$ RP-18 Spheri $5, \mathrm{C}-18,5 \mu \mathrm{m}, \mathrm{LC}$ column) which had been adjusted to a flow rate of $2 \mathrm{~mL} / \mathrm{min}$ of mobile phase (deionized water: acetonitrile: methanol, $45: 10: 100$ ). To each liter of mobile phase was added $100 \mu \mathrm{l}$ of nitric acid (Fisher Scientific, Fair Lawn, NJ) and $0.3 \mathrm{~g}$ potassium bromide (Sigma Chemical Co., St. Louis, MO). Using these conditions the approximate retention times $(\mathrm{min})$ for the aflatoxins are : $\mathrm{B}_{1}=7.6$, $\mathrm{B}_{2}=5.8, \mathrm{G}_{1}=5.1$ and $\mathrm{G}_{2}=3.9$. The limit of detection of the detector at $360 \mathrm{~nm}$ excitation and $440 \mathrm{~nm}$ emission was $5 \mathrm{pg}$. 


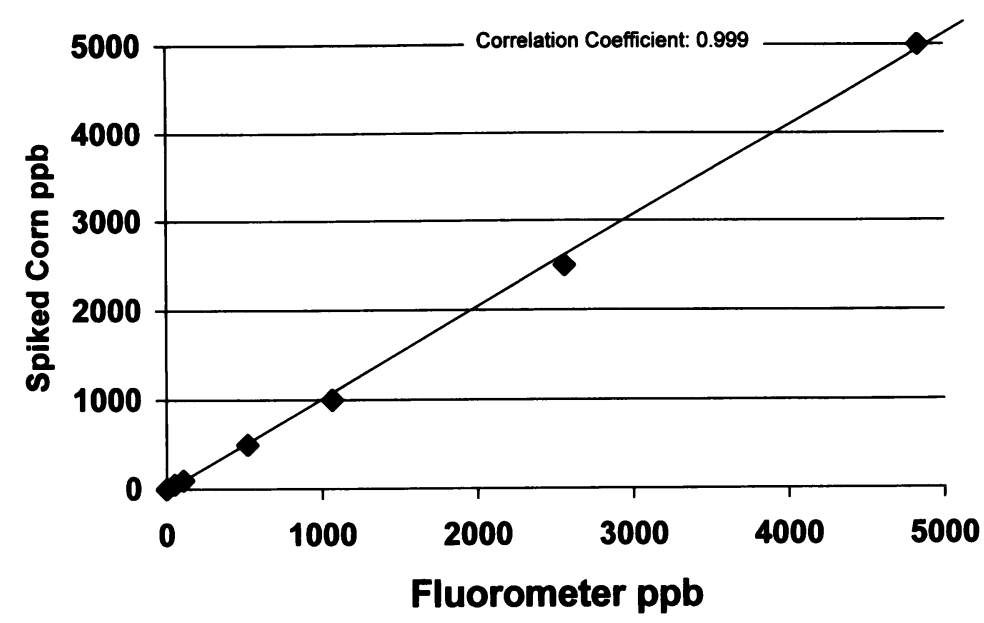

Fig. 1 Linearity of fluorometric measurement of derivatized aflatoxin $B_{1}$ in corn spiked at $0,50,100$, $500,1000,2500$ and $5000 \mathrm{ng} / \mathrm{g}$.

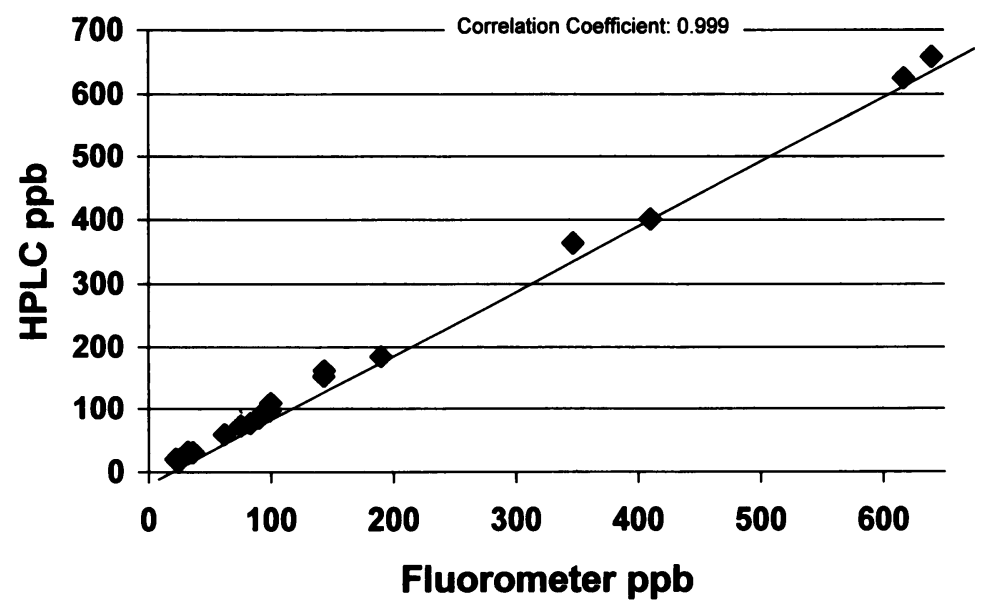

Fig. 2 Fluorometric measurement of total aflatoxin compared to LC analysis in 34 naturally contaminated corn samples. Correlation coefficient $=0.999$.

\section{Results and Discussion}

To examine the procedure for linearity, seven samples of corn containing no detectable aflatoxin were spiked at $0,50,100,500,1000,2500$ and $5000 \mathrm{ng}$ aflatoxin $\mathrm{B}_{1} / \mathrm{g}$ and tested. The results were plotted versus the actual values of the spike (Fig. 1). All spiked samples tested very close to the actual values. The linearity from 0 to $5000 \mathrm{ppb}$ using this method is sufficient to determine any significant levels of naturally occurring aflatoxin in grains and some grain products as well as raw peanuts.

The results of testing thirty four samples of naturally contaminated corn by this fluor- 


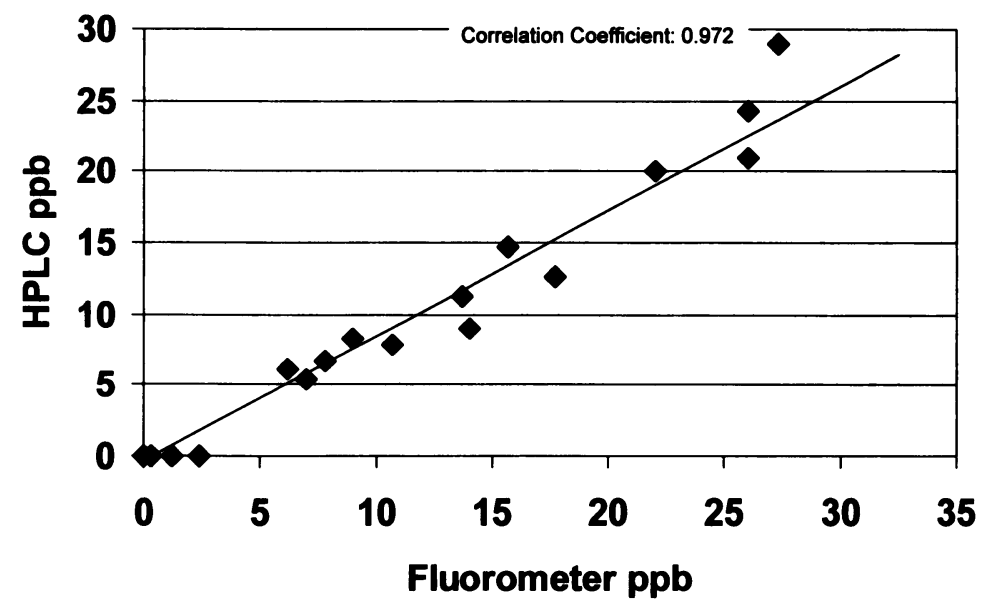

Fig. 3 Fluorometric measurement of total aflatoxin compared to LC analysis in 19 of the 34 corn samples from Figure 3 that had less than $40 \mathrm{ppb}$ of aflatoxin $\mathrm{B}_{1}$. Correlation coefficient $=$ 0.970 .

ometric procedure were very close to those of LC (correlation coefficient $=0.999$ ) applied to the same samples (Fig. 2). When nineteen of the thirty four samples that were below $40 \mathrm{ppb}$ were statistically evaluated the correlation coefficient for these was 0.970 (Fig. 3). Similar correlation coefficients were obtained when thirty two cottonseed samples and eleven peanut paste samples were analyzed by the same two procedures. The test procedure appears to work quite well for sorghum as we have analyzed over 15 different varieties of sorghum without problems.

The entire fluorometric procedure for a single sample took less than 5 minutes to conduct however, multiple samples can be processed at the same time up to the point of addition of the bromine. Once the bromine has been added the sample must be placed in the fluorometer immediately after mixing. Because the fluorometric procedure is rapid and grouping of samples is not required, it can be applied to individual truckloads of grain arriving at buying stations or terminals. Thus, delays for unloading trucks at terminals caused by the time for analysis can be decreased significantly. Furthermore, the quantitative results from this test are obtained as quickly as other qualitative or semi-quantitative tests.

In studies by Cole and Dorner ${ }^{4}$, concerning the extraction efficiency of aflatoxins from peanuts, methanol : water at $80: 20$ was one of the better solvent systems for this matrix. As they noted the solvent system for extraction must be compatible with the analytical system used. In the method reported herein this incompatibility of the solvent system with the SolSep columns was alleviated by adding $1 \mathrm{~mL}$ of acetonitrile to $1 \mathrm{~mL}$ of the extract before pushing the extract through the column matrix.

As noted in similar methods for quantitation of deoxynivalenol ${ }^{5)}$, the use of the pint jar for extraction/blending was required to get adequate mixing of the entire sample during the extraction. Larger vessels allowed for sample material to splash and cling to areas of the vessel not washed by the solvent, thus, extraction was incomplete.

The SolSep \# 2001 and 2002 columns for cleanup proved to be sufficient for analysis of aflatoxin in the specified range of commodities tested and eliminated background fluorescen- 
Table 1 Accuracy and precision for the determinationa of aflatoxin in spiked corn by a fluorometric method.

\begin{tabular}{lcccc}
\hline \hline Spiked Level (ppb) & 10 & 20 & 40 & 160 \\
No. of Replicates & 90 & 90 & 90 & $89^{\mathrm{b}}$ \\
Range (ppb) & $6.5-14$ & $14-25$ & $32-47$ & $130-170$ \\
Mean (ppb) & 10.2 & 19.8 & 38.2 & 147 \\
Recovery & $102 \%$ & $99 \%$ & $96 \%$ & $92 \%$ \\
Standard Deviation & 2.0 & 2.5 & 4.4 & 13 \\
Relative Std. Dev. & $19.6 \%$ & $12.6 \%$ & $11.5 \%$ & $8.8 \%$ \\
\hline
\end{tabular}

a) 3 analysts each using 2 different fluorometers.

b) one outlier.

ce that normally exists in some of the extracted substrates. Also, the column packing material is of such constituency that allows for a prolonged shelf life and no refrigeration is necessary.

Of importance in the fluorometric procedure is the necessity of using fresh bromine daily. To keep the bromine fresh, the appropriate volumes were kept in sealed amber vials for preparing the developer working reagent and the latter was dispensed from amber, repipettor dispensers set for $1 \mathrm{~mL}$ of delivery. We found that the developer working reagent usually was not dependable after $8 \mathrm{hr}$ of use.

The stability of the fluorometer was quite good and normally was calibrated only at the beginning of the daily usage. However, it should be periodically checked during continuous day long usage by inserting the control calibrator to make sure the correct reading is obtained. If the reading is not within the tolerance limits then the machine should be recalibrated.

This method has proved to be safe, reliable, rapid and accurate (Table 1) and has been applied to a number of commodities successfully. However, it should be applied only to those commodities that have been included in the studies as each commodity requires the appropriate programming. of the fluorometer for calibration. The correct programs for each commodity are included with the fluorometer.

\section{References}

1) International Agency for Research on Cancer. (1987) IARC monograph on the evaluation of carcinogenic risk to humans. Suppl. 1, IARC, Lyon, France. pp. 82-87.

2) Trucksess, M. W. \& Wood, G. E. (1994) Recent methods of analysis of aflatoxins in foods and feeds. IN : The Toxicology of Aflatoxins, Human Health, Veterinary and Agricultural Significance, Eaton, D. L. \& Groopman, J. D. Eds., Academic Press, Inc., New York, pp. 409-431.

3) Kok, W. Th., vanNeer, C. H.Th., Traag, W. A. \& Tuinstra, L. B. M. Th. (1986) Determinatioon of aflatoxins in cattle feed by liquid chromatography and post-column derivatization with electrochemically generated bromide. J. Chromato. 367, 231-236.

4) Cole, R. J. \& Dorner, J. W. (1994) Extraction of aflatoxins from naturally contaminated peanuts with different solvents and solvent/peanut ratios. J. AOAC, Intnl., 77, 1509-1511.

5) Malone, B. R., Humphrey, C. W., Romer, T. R. \& Richard, J. L. (1998) One-step solid-phase extraction and cleanup and fluorometric analysis of deoxynivalenol in grains. J. AOAC, Intnl., 81 : $448-452$ 